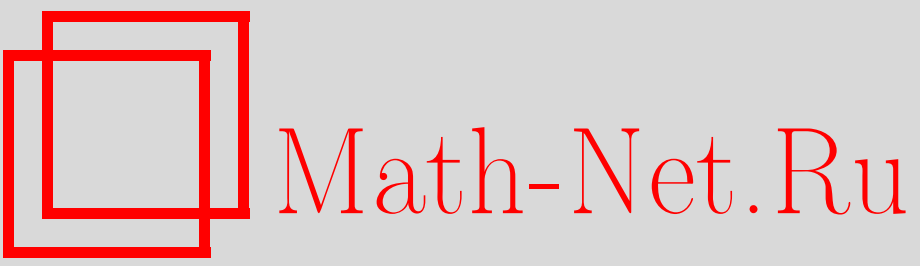

М. Е. Перес, Г. А. Чечкин, Е. И. Яблокова, О собственных колебаниях тела с “легкими” концентрированными массами на поверхности, УМH, 2002, том 57, выпуск 6, 195-196

DOI: https://doi.org/10.4213/rm589

Использование Общероссийского математического портала Math-Net.Ru подразумевает, что вы прочитали и согласны с пользовательским соглашением

http://www. mathnet.ru/rus/agreement

Параметры загрузки:

IP : 54.197 .217 .227

26 апреля 2023 г., 11:56:34 


\title{
О СОБСТВЕННЫХ КОЛЕБАНИЯХ ТЕЛА С “ЛЕГКИМИ" КОНЦЕНТРИРОВАННЫМИ МАССАМИ НА ПОВЕРХНОСТИ
}

\author{
М. Е. ПЕРес, Г. А. ЧЕчКин, Е. И. ЯБЛоковА (ДоронинА)
}

Постановка задачи. Мы рассматриваем спектральную задачу для оператора Лапласа с быстро меняющимся типом граничных условий в области с большим количеством концентрированных масс около границы. Не предполагая периодичности микроструктуры области, авторы рассматривают случай, когда плотность концентрированных масс невелика.

В статьях [1] и [2] рассматриваются похожие задачи в областях с концентрированными массами, расположенными периодически вдоль границы; в [3] изучается задача с произвольной структурой распределения граничных условий в областях с постоянной плотностью.

Пусть $\Omega \subset \mathbb{R}^{n}$ - ограниченная область с гладкой границей, $n \geqslant 3$. Полагаем, что $\partial \Omega=$ $\bar{\Gamma}_{\varepsilon} \cup \bar{\gamma}_{\varepsilon}$, при этом $\Gamma_{\varepsilon}=\bigcup_{k=1}^{N_{\varepsilon}} \Gamma_{\varepsilon}^{k}$, где $\operatorname{diam}\left(\Gamma_{\varepsilon}^{k}\right) \leqslant 2 \varepsilon$, а расстояние между двумя множествами $\Gamma_{\varepsilon}^{k}$ больше или равно $2 \varepsilon ; \varepsilon-$ малый положительный параметр.

Рассмотрим краевую задачу

$$
-\Delta u_{\varepsilon}+\beta \rho_{\varepsilon}(x) u_{\varepsilon}=\rho_{\varepsilon}(x) f(x) \quad \text { в } \Omega, \quad u_{\varepsilon}=0 \quad \text { на } \Gamma_{\varepsilon}, \quad \frac{\partial u_{\varepsilon}}{\partial \nu}+a(x) u_{\varepsilon}=0 \quad \text { на } \gamma_{\varepsilon},
$$

где $f \in L_{2, \rho_{\varepsilon}}(\Omega)$ (пространство с нормой $\left.\|f\|_{L_{2, \rho_{\varepsilon}}(\Omega)}^{2} \equiv \int_{\Omega} f^{2}(x) \rho_{\varepsilon}(x) d x\right)$, ограниченная измеримая функция $a(x) \geqslant 0$, константа $\beta \geqslant 0, \nu=\left(\nu_{1}, \nu_{2}, \ldots, \nu_{n}\right)$ - внешняя нормаль к границе.

Мы рассматриваем плотность вида $\rho_{\varepsilon}(x)=1$ в $\Omega \backslash \overline{B_{\varepsilon}}, \rho_{\varepsilon}(x)=\varepsilon^{-m}$ в $\Omega \cap B_{\varepsilon}$, где $B_{\varepsilon}=$ $\bigcup_{k=1}^{N_{\varepsilon}} B_{\varepsilon}^{k}$ и $B_{\varepsilon}^{k}=\left\{\left(x_{1}-\xi_{1}^{k}\right)^{2}+\cdots+\left(x_{n}-\xi_{n}^{k}\right)^{2}<\varepsilon^{2}\right\}$, здесь $\left(\xi_{1}^{k}, \ldots, \xi_{n}^{k}\right)=p_{\varepsilon}^{k} \in \Gamma_{\varepsilon}^{k}$, $k=1, \ldots, N_{\varepsilon}$, и $\overline{B_{\varepsilon}^{k}} \cap \partial \Omega \subset \Gamma_{\varepsilon}^{k}$.

Множества $B_{\varepsilon}^{k}$ назовем концентрированными массами. Мыпредполагаем, что плотность концентрированных масс не слишком велика, т.е. $0 \leqslant m<2$.

Как хорошо известно, для каждого фиксированного $\varepsilon$ задача (1) имеет единственное слабое решение $u_{\varepsilon}$ в $H^{1}\left(\Omega, \Gamma_{\varepsilon}\right)$, где $H^{1}\left(\Omega, \Gamma_{\varepsilon}\right)$ мш определим как замькание по норме $H^{1}(\Omega)$ множества функций $C^{\infty}(\bar{\Omega})$, равных нулю в окрестности $\Gamma_{\varepsilon}$.

Пусть $u(x)$ - слабое решение в $H^{1}(\Omega)$ следующей задачи:

$$
-\Delta u+\beta u=f(x) \quad \text { в } \Omega, \quad \frac{\partial u(x)}{\partial \nu}+a(x) u(x)=0 \quad \text { на } \partial \Omega .
$$

Если $\beta=0$ и $a(x) \equiv 0$, то для разрешимости предельной задачи с самого начала предполагается, что $\int_{\Omega} f(x) d x=0$. В этом случае выберем решение $u \in H^{1}(\Omega)$ задачи $(2)$, удовлетворяющее условию $\langle u\rangle \equiv \frac{1}{|\Omega|} \int_{\Omega} u_{\varepsilon}(x) d x=0$.

Оценки. В зависимости от $a(x), \beta$ и числа концентрированных масс $N_{\varepsilon}$, мы получаем равномерную ограниченность по $\varepsilon$ решения $u_{\varepsilon}$ задачи $(1)$, решения предельной задачи, а также разности решений $u_{\varepsilon}-u$ (или, в случае $\beta=0$ и $a(x) \equiv 0$, разности $\left.u_{\varepsilon}-\left\langle u_{\varepsilon}\right\rangle-u\right)$. Для этой цели нам необходимо ввести некоторую специалную срезающую функцию $\psi_{\varepsilon}$. Пусть $\psi(s) \in C^{\infty}\left(\mathbb{R}^{1}\right)$, $0 \leqslant \psi \leqslant 1 \forall s \in \mathbb{R}^{1}, \psi(s)=0$ при $|s| \leqslant 1$ и $\psi(s)=1$ при $|s|>1+\sigma$, где $0<\sigma<1 / 2$.

Определим $\psi_{\varepsilon}=\prod_{k=1}^{N_{\varepsilon}} \psi_{\varepsilon}^{k} \mathrm{c} \psi_{\varepsilon}^{k}:=\psi\left(|\ln \varepsilon| /\left|\ln r_{k}\right|\right)$, где $\left(r_{k}, \theta_{k}^{1}, \theta_{k}^{2}, \ldots, \theta_{k}^{n-1}\right)$ - локальная система полярных координат с центром в точке $p_{\varepsilon}^{k} \in \Gamma_{\varepsilon}^{k}, k=1, \ldots, N_{\varepsilon}$.

Теорема 1. Пусть $\beta$ и а(x) одновременно не равны нулю. Пусть $N_{\varepsilon}=O\left(|\ln \varepsilon|^{\alpha}\right) c$ $0<\alpha<1-1 / n$. Для решения иє задачи (1) и решения и задачи (2) имеем

- $\left\|u_{\varepsilon}\right\|_{H^{1}(\Omega)} \leqslant C_{1}\|f\|_{L_{2, \rho_{\varepsilon}}(\Omega)}$

- $\left\|\left(u_{\varepsilon}-u\right) \psi_{\varepsilon}\right\|_{H^{1}(\Omega)} \leqslant C_{2}\|f\|_{L_{2, \rho_{\varepsilon}}(\Omega)}|\ln \varepsilon|^{\alpha+\frac{1}{n}-1}$;

- $\left\|\left(u_{\varepsilon}-u\right)\left(1-\psi_{\varepsilon}\right)\right\|_{L^{2}(\Omega)} \leqslant C_{3^{\varepsilon}} \frac{1}{1+\sigma}|\ln \varepsilon|^{\frac{\alpha}{2}}\|f\|_{L_{2, \rho_{\varepsilon}}(\Omega)}$; 
- если $m=0$ u $f \in L_{2}(\Omega)$, то $u_{\varepsilon} \rightarrow$ сллабо сходится в $H^{1}(\Omega)$ при $\varepsilon \rightarrow 0$;

- если $0<m<2$ uf $f H^{1}(\Omega)$, mо $u_{\varepsilon} \rightarrow$ сллабо сходится в $H^{1}(\Omega)$ при $\varepsilon \rightarrow 0$; $C_{1}, C_{2}$ и $C_{3}$ - константы, не зависящие от $\varepsilon$.

Teоpema 2. Пусть $a(x) \equiv 0, \beta=0 u N_{\varepsilon}=O\left(|\ln \varepsilon|^{\alpha}\right) c 0<\alpha<\min (1-1 / n, 2-m)$. Для решения иє задачи (1) и решения и задачи (2) имеем

- $\left\|u_{\varepsilon}-\left\langle u_{\varepsilon}\right\rangle\right\|_{H^{1}(\Omega)} \leqslant C_{4}\|f\|_{L_{2, \rho_{\varepsilon}}(\Omega)}$;

- $\left\|\left(u_{\varepsilon}-\left\langle u_{\varepsilon}\right\rangle-u\right) \psi_{\varepsilon}\right\|_{H^{1}(\Omega)} \leqslant C_{5}\|f\|_{L_{2, \rho_{\varepsilon}}(\Omega)}|\ln \varepsilon|^{\alpha+\frac{1}{n}-1}$;

- $\left\|\left(u_{\varepsilon}-\left\langle u_{\varepsilon}\right\rangle-u\right)\left(1-\psi_{\varepsilon}\right)\right\|_{L^{2}(\Omega)} \leqslant C_{6} \varepsilon^{\frac{1}{1+\sigma}}|\ln \varepsilon|^{\frac{\alpha}{2}}\|f\|_{L_{2, \rho_{\varepsilon}}(\Omega)}$;

- если $m=0$ u $f \in L_{2}(\Omega)$, то $u_{\varepsilon}-\left\langle u_{\varepsilon}\right\rangle \rightarrow$ u слабо сходится в $H^{1}(\Omega)$ при $\varepsilon \rightarrow 0$;

- если $0<m<2 u f \in H^{1}(\Omega)$, то $u_{\varepsilon}-\left\langle u_{\varepsilon}\right\rangle \rightarrow u$ слабо сходится в $H^{1}(\Omega)$ nри $\varepsilon \rightarrow 0$

$C_{4}, C_{5}$ и $C_{6}$ - константы, не зависящие от $\varepsilon$.

Асимптотика собственных значений. Рассмотрим спектральные задачи, соответствующие краевым задачам (1) и (2):

$$
\Delta\left(u_{\varepsilon}^{k}\right)+\lambda_{\varepsilon}^{k} \rho_{\varepsilon}(x) u_{\varepsilon}^{k}=0 \text { в } \Omega, \quad u_{\varepsilon}^{k}=0 \text { на } \Gamma_{\varepsilon}, \quad \frac{\partial u_{\varepsilon}^{k}}{\partial \nu}+a(x) u_{\varepsilon}^{k}=0 \text { на } \gamma_{\varepsilon}, k=1,2, \ldots,
$$
и, соответственно,

$$
\Delta\left(u^{k}\right)+\lambda_{0}^{k} u^{k}=0 \quad \text { в } \Omega, \quad \frac{\partial u^{k}}{\partial \nu}+a(x) u^{k}=0 \quad \text { на } \partial \Omega, \quad k=1,2, \ldots
$$

Здесь $u_{\varepsilon}^{k} \in H^{1}\left(\Omega, \Gamma_{\varepsilon}\right), u^{k} \in H^{1}(\Omega), k=1,2, \ldots$, образуют ортогональньй базис в $L_{2, \rho_{\varepsilon}}(\Omega)$ и $L_{2}(\Omega)$ соответственно, а $\left\{\lambda_{\varepsilon}^{k}\right\},\left\{\lambda_{0}^{k}\right\}, k=1,2, \ldots,-$ множества соответствующих собственных значений, занумерованных в порядке неубывания $0<\lambda_{\varepsilon}^{1} \leqslant \lambda_{\varepsilon}^{2} \leqslant \cdots \leqslant \lambda_{\varepsilon}^{k} \leqslant \cdots, 0 \leqslant \lambda_{0}^{1} \leqslant$ $\lambda_{0}^{2} \leqslant \cdots \leqslant \lambda_{0}^{k} \leqslant \cdots$ и повторяющихся в соответствии с их кратностью $\left(\lambda_{0}^{1}>0\right.$, если $\left.a(x) \not \equiv 0\right)$.

Используя спектральную теорему о предельном поведении спектра положительного самосопряженного компактного оператора, определенного на гильбертовом пространстве, зависящем от малого параметра (см. теоремы 1.9 и 1.12 главы III в [4]), мы получаем оценки для разности собственных элементов задач (3) и (4).

ТЕорема 3. Для собственных значений $\lambda_{\varepsilon}^{k}, \lambda_{0}^{k}$ задач (3) и (4), соответственно, имеет место оченка $\left|\lambda_{\varepsilon}^{k}-\lambda_{0}^{k}\right| \leqslant A_{k}|\ln \varepsilon|^{\alpha+\frac{1}{n}-1}$ при достаточно малом $\varepsilon$, где $N_{\varepsilon}=$ $O\left(|\ln \varepsilon|^{\alpha}\right), 0<\alpha<1-1 / n, \varepsilon \rightarrow 0$, и для каждого $k=1,2, \ldots$ константы $A_{k}$ не зависят om $\varepsilon$.

Теорема 4. Рассмотрим условия теоремь 3. Предположим, что $k, l$ целье, $k \geqslant 1$, $l \geqslant 1$, и $\lambda_{0}^{k}<\lambda_{0}^{k+1}=\cdots=\lambda_{0}^{k+l}<\lambda_{0}^{k+l+1}$. Тогда для любой собственной функции $w$ задачи (4), соответствующей собственному значению $\lambda_{0}^{k+1}$, существует линейная комбинация $\bar{u}_{\varepsilon}$ собственных функций $u_{\varepsilon}^{k+1}, \ldots, u_{\varepsilon}^{k+l}$ задачи (3) такая, что $\| \bar{u}_{\varepsilon}-$ $R_{\varepsilon} w \|_{H_{\varepsilon}} \leqslant B_{k}|\ln \varepsilon|^{\alpha+\frac{1}{n}-1}$, әде константы $B_{k}$ не зависят от $\varepsilon$.

\section{СПИСОК ЛИТЕРАТУРЫ}

[1] Г. А. Чечкин // УМН. 1995. Т. 50. № 4. С. 105-106. [2] M. Lobo, M. E. Pérez // Math. Models Methods Appl. Sci. 1993. V. 3. № 2. P. 249-273. [3] O. A. Oleinik, G. A. Chechkin // Atti Accad. Naz. Lincei Cl. Sci. Fis. Mat. Natur. Rend. Lincei (9) Mat. Appl. 1996. V. 7. № 1. Р. 5-15. [4] О.А. Олейник, Г.А. Иосифьян, А. С. Шамаев. Математические задачи теории сильно неоднородных упругих сред. М.: Изд-во МГУ, 1990. 